\title{
Efetividade de um Programa Multiprofissional de Tratamento da Obesidade em adolescentes: impacto sobre transtorno de compulsão alimentar periódica
}

\author{
Nelson Nardo Júnior ${ }^{1}$, Marciele Alves Bolognese ${ }^{1}$, Josiane Aparecida Alves Bianchini ${ }^{1}$, Danilo \\ Fernandes da Silva ${ }^{1}$, Anselmo Alexandre Mendes ${ }^{1}$ e Rafaela Pilegi Dada ${ }^{1}$
}

Os objetivos deste estudo foram avaliar os efeitos de um Programa Multiprofissional de Tratamento da Obesidade (PMTO-NEMO-UEM) sobre o transtorno de compulsão alimentar periódica (TCAP) em adolescentes; verificar o impacto do sexo e faixa etária sobre as mudanças no escore de compulsão alimentar e; relacionar as mudanças percentuais neste escore com as mudanças percentuais nas variáveis antropométricas e composição corporal. A amostra foi composta por 27 adolescentes com excesso de peso entre 14 e 17 anos, que passaram por avaliações antes e após um PMTO composto por nutricionistas, educadores físicos e uma psicóloga. Foram obtidas as medidas de peso, índice de massa corporal (IMC), composição corporal, e TCAP, a partir da escala de compulsão alimentar periódica. Verificou-se que o PMTO-NEMO-UEM foi capaz de reduzir os escores de compulsão alimentar em adolescentes, independente do sexo e faixa etária, além de promover melhoras sobre o peso, IMC, gordura corporal, e massa livre de gordura relativa. O programa também reduziu o número de adolescentes com TCAP após o período de intervenção (18,5\% versus 3,7\%). Assim, conclui-se que um PMTO pode promover mudanças positivas sobre os escores de compulsão alimentar em adolescentes, sendo uma possibilidade de serviço de saúde voltado à população pediátrica.

Palavras-chave: efetividade, intervenção, obesidade, transtorno de compulsão alimentar.

\section{Effectiveness of a Multiprofessional Program of Obesity Treatment in adolescents: impact on binge eating disorder}

The objectives of this study were assess the effects of a Multiprofessional Program of Obesity Treatment (PMTONEMO-UEM) on the binge eating disorder (TCAP) in adolescents; verify the impact of gender and age on the rates of binge eating and correlate percentage changes in this score with percentage changes in anthropometric parameters and body composition. The sample was composed by 27 adolescents with excess body weight, aged between 14 and 17 years, who were subjected to assessments before and after a PMTO composed by nutritionists, physical educators, and a psychologist. The parameters body weight, body mass index (BMI), body composition, and TCAP were assessed, based on the binge eating disorder scale. PMTO-NEMO-UEM was able to reduce the score of binge eating in adolescents, regardless of gender and age, besides promoting improvements in body weight, BMI, body fat, and relative fat-free mass. The program also decreased the rates of binge eating in

${ }^{1}$ Núcleo de Estudos Multiprofissional da Obesidade, Departamento de Educação Física, Universidade Estadual de Maringá, Maringá, PR, Brasil. Endereço para correspondência: Avenida Colombo no 5.790, Jardim Universitário, Bloco 05, Sala 4, CEP 87020-900, Maringá, PR, Brasil. Telefone para contato: (44)30115026 - Fax: (44)30263988. E-mail: nnjunior@uem.br 
adolescents after the intervention period (18.5\% versus $3.7 \%)$. Thus, it is concluded that PMTO can improve binge eating disorder scale in adolescents, being an alternative as a health care service focused on the pediatric population.

Keywords: effectiveness, intervention, obesity, binge eating disorder.

\section{INTRODUÇÃO}

O cenário atual do Brasil em relação à prevalência de excesso de peso é preocupante. Pesquisas atuais demonstram que o excesso de peso na população infanto-juvenil tem aumentado no Brasil [1,2]. Resultados de uma meta-análise realizada com 21 estudos e amostra de 18.463 crianças e adolescentes apontam que a prevalência de obesidade foi de $14,1 \%{ }^{[3]}$. Assim, a implementação de intervenções para o tratamento da obesidade e suas comorbidades são urgentes para o controle da situação, prevenção de consequências associadas à doença e melhora da saúde de crianças e adolescentes [3,4].

Documentos e guias nacionais e internacionais para o tratamento da obesidadesão unânimes em orientar que o tratamento convencional deve ser sempre o primeiro passo, por não oferecer riscos e efeitos colaterais que podem estar relacionados aos métodos não convencionais, como o uso de medicamentos e cirurgia bariátrica especialmente na fase da adolescência [47].

Segundo Cubrelati [8], durante a adolescência imagina-se um corpo ideal que, no entanto, nem sempre corresponde ao corpo real, e quanto mais o corpo real estiver longe do ideal, maior será a frustração e redução da autoestima ligada a uma distorção de imagem corporal. Essa distorção da imagem corporal em conjunto com a baixa autoestima, é responsável pela busca incessante por emagrecimento, levando a comportamentos prejudiciais à saúde, tais como o transtorno de compulsão alimentar periódica (TCAP).

O TCAP pode ser caracterizado pela presença de ao menos três dos seguintes comportamentos: comer muito e mais rápido que o habitual, comer até sentir-se cheio de modo a incomodar, alimentar-se com grandes quantidades de comida mesmo sem estar com fome, realizar as refeições sozinho ou escondido por se sentir envergonhado pela grande quantidade de comida ingerida e sentir repulsa ou raiva de si mesmo após algum episódio de compulsão alimentar ${ }^{[9,10]}$.
No Brasil, entre 15 e $22 \%$ dos pacientes que buscam programas de emagrecimento apresentam TCAP, e essa prevalência pode ser ainda maior na população obesa (cerca de 30\%) [11]. Entretanto, até onde se tem conhecimento, ainda não há estudos verificando os efeitos de intervenções convencionais (e.g., programas multiprofissionais) sobre os escores da escala de compulsão alimentar periódica, sobretudo em adolescentes. Assim, os objetivos do presente estudo foram: a) avaliar os efeitos de um Programa Multiprofissional de Tratamento da Obesidade (PMTONEMO-UEM) sobre o TCAP em adolescentes; b) verificar o impacto das variáveis sexo e faixa etária do adolescente sobre as mudanças obtidas no escore de compulsão alimentar; c) relacionar as mudanças percentuais no escore de compulsão alimentar com as mudanças percentuais dos valores dass variáveis antropométricas e composição corporal.

\section{MATERIAL E MÉTODOS}

\section{Caracterização do estudo}

Trata-se de um estudo piloto, caracterizado como ensaio clínico pragmático, desenhado para avaliar a efetividade das intervenções em condições práticas da vida real. Apesar da desvantagem da não randomização em cada grupo, ensaios pragmáticos produzem resultados que podem ser generalizados e aplicados na rotina dos indivíduos [12].

\section{Programa Multiprofissional de Tratamento da Obesidade (PMTO)}

O projeto de atendimento a adolescentes com excesso de peso oferecido pelo Núcleo de Estudos Multiprofissional da Obesidade (NEMO), da Universidade Estadual de Maringá teve seu início no segundo semestre de 2005. Até o ano de 2008, diferentes profissionais da saúde contribuíram com o atendimento à comunidade, entre eles, profissionais de educação física, nutrição, psicologia, enfermagem, odontologia, fisioterapia, fonoaudiologia e medicina. 
A partir de 2009, o PMTO-NEMO-UEM se consolidou como equipe, contando com a participação de profissionais e estudantes de educação física, nutrição, psicologia e medicina (pediatria) que passaram a atuar com base na terapia cognitivo comportamental [13]. O modelo atual, conta com as áreas de nutrição, educação física e psicologia. Este modelo de intervenção foi aprovado pelo Comitê de Ética local (protocolo 451.764/2013). Até o momento, 164 adolescentes já ingressaram no grupo intervenção, com idade variando entre 10 e 18 anos. Destes, 61 (37,2\%) não completaram ao menos $70 \%$ da intervenção e/ou não concluíram o período de intervenção/avaliações. Os motivos de desistência relatados pelos adolescentes foram: questões de transporte, desmotivação para continuar na intervenção e preferência por outras atividades no mesmo período.

O principal objetivo do programa é contribuir com mudanças positivas em variáveis da composição corporal, em médio prazo, a partir do incentivo e prática de atividade física e orientação de hábitos saudáveis relacionados à alimentação. Previamente, foi demonstrado o efeito positivo desse modelo de intervenção sobre variáveis da composição corporal e aptidão física ${ }^{[14]}$, perfil hemodinâmico, lipídico e resistência à insulina [15]. Trabalhos em fase de revisão mostram os resultados desse modelo de intervenção sobre frequência alimentar, hábitos alimentares e qualidade de vida relacionada à saúde (QVRS).

Para o presente estudo em específico, o PMTO-NEMO-UEM foi focado em adolescentes com idade entre 14 e 17 anos, tendo em vista a necessidade da obtenção de resultados de programas convencionais, em especial de caráter multiprofissional, mais direcionados a idades mais avançadas durante a adolescência, que segundo a Portaria 425/2013 lançada recentemente, já teriam condições de serem submetidos à cirurgia bariátrica pelo Sistema Único de Saúde. Foram realizadas divulgações na mídia local e regional convidando jovens nessa faixa etária para participarem do PMTO-NEMO-UEM. Em reunião agendada com as familias dos adolescentes interessados em fazer parte do projeto foi explicado, em detalhes, os objetivos e os tipos de intervenções aos quais os mesmos se submeteriam, bem como sobre a liberdade de deixar de participar a qualquer momento se desejassem. Com aqueles que inicialmente concordaram em participar, foi firmado um termo de compromisso livre e esclarecido, no qual todas estas informações estavam explicitadas. Foram adotados os seguintes critérios de inclusão para formação do grupo intervenção: apresentar sobrepeso ou obesidade de acordo com os pontos de corte propostos por Cole e Lobstein [16] baseados na idade e sexo; concordância do adolescente e seu responsável em participar do PMTO-NEMO-UEM e disponibilidade para participar integralmente das intervenções. Os critérios de exclusão foram: problemas genéticos, endócrinos ou metabólicos que requeriam o uso de quaisquer medicamentos que pudessem interferir no controle do excesso de gordura corporal (exemplo: uso de glicocorticoides ou psicotrópicos) e que foram previamente diagnosticados e informados ao pediatra; consumo em longo prazo de álcool; taxa de adesão de, no mínimo, $70 \%$ das intervenções multiprofissionais [17].

O PMTO-NEMO-UEM foi realizado durante 16 semanas, com intervenções três vezes na semana (segundas, quartas e sextas feiras), sendo a primeira parte destinada às intervenções práticas da educação física que tem duração de uma hora a uma hora e meia. A segunda parte dos três dias da semana foi destinada à intervenção teórica da educação física, nutrição e psicologia.

\section{Protocolo de intervenção}

As intervenções teóricas oferecidas nesse período aconteceram três vezes na semana, com duração média de uma hora, abordando as áreas da psicologia, nutrição e educação física.

As intervenções da área de psicologia abordaram os seguintes tópicos: a) estabelecimento de metas; b) auto-observação dos próprios comportamentos e consequências (imediatas, médio prazo e longo prazo); c) discriminação de sensações e análise de sentimentos; d) imagem corporal; e) ampliação do autoconhecimento e análise dos eventos internos (pensamentos) e suas consequências; f) aspectos relacionados à automotivação e autocontrole; g) relacionamentos interpessoais (habilidades sociais). Sendo conduzia por uma psicóloga especialista em terapia cognitivo comportamental e uma acadêmica do curso de psicologia, por meio de reuniões semanais em grupo com duração média de uma hora.

As intervenções nutricionais tiveram como objetivo orientar sobre aspectos nutricionais, tais como: a) pirâmide alimentar; b) densidade energética dos alimentos; c) importância dos macro e micronutrientes e sua relação com a saúde; d) composição nutricional dos alimentos; dentre outros, aconteceram intervenções na área de nutrição, com uma nutricionista experiente. 
Foram realizadas intervenções semanais, conduzidas pelos profissionais e acadêmicos de Educação Física, a fim de fornecer informações e esclarecimentos sobre a prática de atividade física e seus benefícios e estimulá-los a praticar atividade física fora do horário de intervenção. Além disso, foi promovida a prática regular de atividade física supervisionada com a mesma duração da parte teórica (uma horaa uma hora e meia) com frequência de três aulas semanais, onde foram executados exercícios em meio aquático e terrestre, priorizando exercícios aeróbios (caminhada/corrida), exercícios resistidos e iniciação esportiva.

\section{Avaliações}

Ao início e fim da intervenção os adolescentes passaram por uma bateria de avaliações no período da tarde, as quais incluíram a medida do peso, realizada por meio de uma balança da marca Welmy com capacidade para medir até $300 \mathrm{~kg}$ e precisão de $0,05 \mathrm{~kg}$ e o Indice de Massa Corporal (IMC), calculado a partir da divisão do peso dos adolescentes pela sua altura ao quadrado.

A composição corporal foi avaliada utilizando a bioimpedância octapolarmultifrequencial, da marca InBody, modelo 520. Os sujeitos foram avaliados com roupas leves e sem portarem nenhum objeto metálico, seguindo as recomendações de Heyward [18]. Foram computadas as variáveis, gordura corporal relativa e absoluta e massa magra relativa e absoluta.

O TCAP foi avaliado a partir da Escala de compulsão alimentar periódica (ECAP), que é constituída por 16 questões. Esta é uma escala autoaplicável e desenvolvida por Gormally et al. [19], sendo adequada para discriminar indivíduos obesos com e sem TCAP, com consistência interna de 0,85 (alfa de Cronbach). $\mathrm{O}$ instrumento é respondido em uma escala indo de 0 (ausência de gravidade) a 3 (gravidade máxima). O escore final é o resultado da soma dos pontos de cada item. Esse instrumento foi adequadamente validado para língua portuguesa por Freitas et al. [20]. Os sujeitos podem ser classificados com e sem TCAP segundo a seguinte pontuação: 0-17 sem TCAP e $>17$ com TCAP [21].

\section{Análise Estatística}

Inicialmente, testou-se a normalidade dos dados por meio do teste de Shapiro-Wilk. Sendo confirmada a normalidade da distribuição dos dados, foi aplicado o teste $t$ para amostras dependentes e quando o pressuposto da normalidade não foi confirmado, fez-se uso do teste de Wilcoxon para comparação pré e pósintervenção. Para associar a frequência de adolescentes com TCAP antes e após o PMTO, aplicou-se o teste de McNemar. A comparação entre meninos e meninas, e entre as faixas etárias de 14-15 anos e 16-17 anos foi elaborada por meio do teste $t$ para amostras independentes. As correlações entre a variação percentual dos escores de compulsão alimentar com a variação percentual das variáveis antropométricas foi feita a partir do coeficiente de correlação de Spearman. Para todas as análises, o nível de significância foi préestabelecido em $p<0,05$.

\section{RESULTADOS}

A Tabela 1 apresenta os resultados dos 27 adolescentes avaliados antes e após 16 semanas do programa de intervenção para os parâmetros antropométricos, composição corporal e o escore obtido pelo questionário de CA. 
Tabela 1. Antropometria, composição corporal e escore de compulsão alimentar antes e após 16 semanas do PMTO (n=27).

\begin{tabular}{lccc}
\hline \multicolumn{1}{c}{ Variáveis } & Pré-intervenção & Pós-intervenção & $p$ \\
\hline Peso $(\mathrm{kg})$ & $91,2 \pm 17,4$ & $85,7 \pm 15,0$ & $<0,001^{*}$ \\
IMC $\left(\mathrm{kg} / \mathrm{m}^{2}\right)^{\#}$ & $29,7(27,4-35,3)$ & $28,0(26,1-32,5)$ & $<0,001^{*}$ \\
Gordura $(\mathrm{kg})$ & $37,5 \pm 11,2$ & $31,2 \pm 10,3$ & $<0,001^{*}$ \\
Gordura $(\%)$ & $40,3 \pm 7,3$ & $36,1 \pm 7,7$ & $<0,001^{*}$ \\
MLG $(\mathrm{kg}) \#$ & $52,8(44,4-63,8)$ & $54,2(45,5-62,9)$ & 0,056 \\
MLG $(\%)$ & $59,3 \pm 6,9$ & $63,9 \pm 7,7$ & $<0,001^{*}$ \\
CA & $13,0 \pm 5,9$ & $6,6 \pm 3,8$ & $<0,001^{*}$ \\
\hline
\end{tabular}

Teste $t$ para amostras dependentes

\#Teste de Wilcoxon

IMC $=$ Índice de Massa Corporal

MLG $=$ Massa Livre de Gordura

$\mathrm{CA}=$ Escore de compulsão alimentar

$* p<0,05$.

Na Tabela 2 estão os resultados do teste de associação entre o número de adolescentes com TCAP antes e após o período de intervenção. Ao iniciar o
PMTO, cinco (18,5\%) adolescentes apresentaram TCAP, enquanto que esse número foi reduzido para apenas um $(3,7 \%)$ adolescente ao final do programa.

Tabela 2. Associação entre presença de TCAP antes e após o PMTO ( $n=27)$.

\begin{tabular}{lccc}
\hline \multirow{2}{*}{ Pós-intervenção } & Baseline & \\
& Com TCAP & Sem TCAP & $p$ \\
\hline Com TCAP & 0 & 1 & 0,219 \\
Sem TCAP & 5 & 21 & \\
\hline TCAP = Transtorno de Compulsão Alimentar Periódica & &
\end{tabular}

$\mathrm{Na}$ Tabela 3 é possível observar que tanto o sexo quanto a faixa etária do adolescente não impactaram os efeitos do PMTO sobre os escores de compulsão alimentar. Ademais, tanto no pré como no pós-intervenção não houve diferenças entre os resultados obtidos para meninos e meninas, assim como entre os adolescentes mais jovens (14-15 anos) e os mais velhos (16-17 anos) ( $p>0,05)$. 
Obesidade em adolescentes e transtorno alimentar. Nardo Júnior $e t$ al.

Tabela 3. Impacto do sexo e faixa etária sobre os escores de compulsão alimentar ( $\mathrm{n}=27)$.

\begin{tabular}{|c|c|c|c|c|c|c|c|}
\hline \multicolumn{4}{|c|}{ Masculino } & \multicolumn{4}{|c|}{ Feminino } \\
\hline Variáveis & Pré-intervenção & Pós-intervenção & $p$ & Variáveis & Pré-intervenção & Pós-intervenção & $p$ \\
\hline CA & $12,7 \pm 5,3$ & $7,3 \pm 3,6$ & 0,004 & CA & $13,2 \pm 6,6$ & $5,9 \pm 3,9$ & $<0,001$ \\
\hline \multicolumn{4}{|c|}{ 14-15 anos } & \multicolumn{4}{|c|}{ 16-17 anos } \\
\hline Variáveis & Pré-intervenção & Pós-intervenção & $p$ & Variáveis & Pré-intervenção & Pós-intervenção & $p$ \\
\hline CA & $14,1 \pm 5,4$ & $7,6 \pm 4,3$ & $<0,001$ & CA & $11,7 \pm 6,4$ & $5,5 \pm 2,8$ & 0,002 \\
\hline
\end{tabular}

A Tabela 4 apresenta os resultados da correlação entre a variação percentual do escore de CA com a variação percentual das variáveis antropométricas e composição corporal.

Tabela 4. Correlação entre a variação percentual do escore de CA com a variação percentual das variáveis antropométricas e composição corporal $(n=27)$.

\begin{tabular}{ccccccc}
\hline TCAP & Variação peso & IMC & Gordura $(\mathrm{kg})$ & Variação $(\%)$ gordura & MLG $(\mathrm{kg})$ & MLG \\
\hline$r$ & 0,237 & 0,176 & 0,192 & 0,217 & 0,086 & $-0,198$ \\
$p$ & 0,234 & 0,380 & 0,337 & 0,277 & 0,670 & 0,321 \\
\hline
\end{tabular}

TCAP $=$ Transtorno de Compulsão Alimentar Periódico

IMC $=$ Índice de Massa Corporal

MLG= Massa Livre de Gordura

\section{DISCUSSÃO}

Os principais achados do presente estudo são que um PMTO foi capaz de proporcionar melhora na prevalência de TCAP em adolescentes, além de mudanças significativas do peso, IMC, gordura corporal absoluta, gordura corporal relativa e massa livre de gordura relativa. Adicionalmente, as análises demonstraram que houve uma redução do número de adolescentes com TCAP antes e após o período de intervenção, passandode cinco $(18,5 \%)$ para apenas um (3,7\%) adolescente ao final do programa, porém sem significância estatística.

Análises complementares apontaram que tanto o sexo quanto a faixa etária do adolescente não impactaram os efeitos do PMTO sobre os escores de compulsão alimentar. Além disso, a variação percentual em variáveis antropométricas e composição corporal não explicaram as mudanças observadas no TCAP após a intervenção.
Cerca de 30\% dos pacientes obesos que buscam programas de redução da obesidade apresentaram TCAP [11]. Em adolescentes, esses escores podem variar entre 18,5 e $24 \%$ [22,23]. No presente estudo, ao iniciar o PMTO, esse valor era de 18,5\%, estando de acordo com a literatura.

Porém, destaca-se a positiva redução para 3,7\% após apenas 16 semanas de intervenção, demonstrando o potencial desse modelo de intervenção para controlar problemas de ordem psicossocial [24]. Apesar das meninas estarem mais predispostas a TCAP comparada aos meninos, provavelmente devido à busca pelo corpo magro de forma mais intensa a partir do que é imposto por aspectos da cultura ocidental [25], no presente estudo, não foram encontradas diferenças entre os sexos.

$\mathrm{O}$ aumento da idade também tem relação direta com uma maior prevalência de casos de TCAP em adolescentes [23,25,26], entretanto, os resultados após a intervenção não foram diferentes de acordo com a faixa 
etária dos adolescentes, reforçando que mesmo aqueles mais próximos da faixa etária adulta (e.g., 16-17 anos), e que seriam o grupo de maior risco, podem apresentar melhoras importantes após um programa de intervenção multiprofissional.

Devido à obesidade ser considerada uma doença multifatorial, as intervenções multiprofissionais têm sido apontadas como importante estratégia para o tratamento da obesidade, inclusive em crianças e adolescentes [4,5]. Esse modelo de intervenção tem demonstrado importantes resultados em diferentes parâmetros de saúde, tais como a melhora de fatores de riscos cardiometabólicos, aptidão cardiorrespiratória, composição corporal, qualidade de vida relacionada à saúde, hábitos alimentares, entre outros [24,27-30].

Estudos anteriores do PMTO-NEMO-UEM demonstram os efeitos positivos de 16 semanas de intervenção multiprofissional nas variáveis antropométricas, composição corporal, aptidão física relacionada à saúde, parâmetros hemodinâmicos e fatores de riscos metabólicos de adolescentes entre 10 e 18 anos [14,15,31,32]. Ademais, estudos em fase de conclusão e processo de avaliação apontam para benefícios nos hábitos alimentares e qualidade de vida relacionada à saúde dos adolescentes (dados não publicados).

Apesar do consenso na literatura em relação aos benefícios de intervenções multiprofissionais para o tratamento da obesidade infanto-juvenil, esse modelo de intervenção no Brasil ainda parece estar mais voltado a iniciativas dentro de Universidades públicas, como por exemplo, os programas desenvolvidos pela Universidade Federal de São Paulo [33], Universidade Estadual Paulista [34], Universidade Federal de Santa Catarina [27], Universidade Federal do Paraná [29] e Universidade Estadual de Maringá, sendo necessária sua expansão como um serviço de saúde pública para beneficiar a demanda da população que necessita desse atendimento.

Considerando que o PMTO-NEMO-UEM é formado por profissionais da área da saúde que já compõem o Programa da Saúde da Família (PSF) e os Núcleos de Apoio à Saúde da Família (NASF), baseado em um método convencional de intervenção para o tratamento da obesidade (sem o uso/gasto com medicamentos e/ou intervenção cirúrgica) e considerando que a prevalência de obesidade na população infanto-juvenil tem crescido ano após ano
${ }^{[1,2]}$ seria importante sua aplicação como um serviço do SUS, em Unidades Básicas de Saúde (UBS) e Centros Esportivos, podendo ser mediado como uma das estratégias do Programa da Saúde da Família (PSF) [35] em conjunto com os Núcleos de Apoio à Saúde da Família (NASF) [36]. Grupos de intervenção para adolescentes obesos poderiam ser formados constantemente, a fim de propor uma alternativa para o controle da obesidade e suas comorbidades associadas.

Em contrapartida, o que temos de concreto, é a Portaria 425 do Ministério da Saúde do Brasil [37], que alterou a idade mínima para a realização da cirurgia bariátrica pelo SUS de 18 para 16 anos de idade, o que levanta a questão sobre a necessidade de se avaliar programas convencionais para essa faixa etária, especialmente sua aplicação e custo-efetividade como serviço de saúde.

O documento da Food and Agriculture Organization (FAO) [38] chama a atenção para a necessidade de intervenções integradas e multidimensionais para uma boa execução das políticas de segurança alimentar e nutricional. De acordo com de Souza [39], apesar dos avanços com a implementação de ações de políticas públicas relacionadas asegurança alimentar e nutricionalno Brasil,ainda há uma ausência de programas específicos de educação alimentar e nutricional voltado para a redução da obesidade por meio de mudanças nos hábitos alimentares (redução no consumo de alimentos com alto teor de sódio, açúcares e gorduras).

O plano de ação (2014-2019) para a prevenção da obesidade em crianças e adolescentes ${ }^{[40]}$ com meta de conter a epidemia de obesidade e contribuir para que as prevalências não continuem aumentando, aponta como estratégia a implementação de um conjunto de políticas, legislação, regulamentações e intervenções efetivas que levarão em conta prioridades e o contexto dos Estados membros. Dentre as estratégias, o documento coloca outras ações multisetoriais que estão relacionadas ao incentivo à prática de atividade física $\mathrm{e}$ hábitos alimentares saudáveis, similares ao PMTONEMO-UEM e outros programas desenvolvidos em outras instituições de ensino superior do país. A partir dos resultados do presente estudo, o programa de intervenção parece promover melhoras importantes sobre o TCAP.

Apesar dos importantes resultados, o presente estudo também apresenta limitações. Por se tratar de 
Obesidade em adolescentes e transtorno alimentar. Nardo Júnior et al.

um estudo piloto, o trabalho apresenta um número amostral pequeno com a ausência do grupo controle. Entretanto, tem como ponto forte oferecer um resultado baseado em um cenário real a partir de um design pragmático que favorece a discussão sobre a aplicação prática desse tipo de intervenção a partir dos resultados obtidos.

\section{CONCLUSÃO}

Em suma, um PMTO pode promover mudanças positivas sobre a prevalência de TCAP em adolescentes. Os resultados trazem consigo importantes implicações práticas e sugerem modelos similares de intervenção entre as possibilidades de programas para redução da obesidade no cenário nacional, em especial na população infanto-juvenil.

\section{AGRADECIMENTO}

Aos membros do Núcleo de Estudos Multiprofissional da Obesidade pela contribuição com a coleta de dados e desenvolvimento da intervenção. À Fundação Araucária, Ministério da Saúde e CAPES pelo suporte financeiro.

\section{REFERÊNCIAS}

[1] Niehues JR, Gonzales AI, Lemos RR, Bezerra PP, Haas P. Prevalence of overweight and obesity in children and adolescents from the age range of 2 to 19 years old in Brazil. Int J Pediatr. (03 jun 2014); 2014:583207. DOI http://www.ncbi.nlm.nih.gov/pmc/articles/PMC4065721/ 10.1155/2014/583207

[2] Ng M, Fleming T, Robinson M, Thomson B, Graetz N, Margono C, et al. Global, regional, and national prevalence of overweight and obesity in children and adults during 19802013: a systematic analysis for the Global Burden of Disease Study 2013. Lancet. 2014;384(9945):766-781.

[3] Nunes MS, Aiello AM, Mello LM, Silva AS, Nunes A. Prevalence of obesity in children and adolescents in Brazil: a meta-analysis of cross-sectional studies. Curr. Pediatr. Rev. 2015;11(1):36-42.

[4] Canadian clinical practice guidelines on the management and prevention of obesity in adults and children. CMAJ. 2007;176(8):1-117.

[5] Task Force U.S. Preventive Services.Childhood Obesity Treatment: Obesity Reduction Strategic Initiative. A Toolkit for Louisiana Primary Care Providers. BMC; Pennington Biomedical Research Center; 2015.

[6] SIGN - Scottish Intercollegiate Guidelines Network. Management of obesity: a national clinical guideline. Edinburgh: SIGH;2010.

[7] ABESO - Associação Brasileira para o estudo da obesidade e da síndrome metabólica. Diretrizes brasileiras de obesidade 2009/2010. 3. ed. Itapevi: AC Farmacêutica; 2009.

[8] Cubrelati BS, Rigoni PAG, Vieira LF, Belem IC. Relação entre distorção de imagem corporal e risco de desenvolvimento de transtornos alimentares em adolescentes. Conexões: Revista da Faculdade de Educação Física da UNICAMP. 2014;12(1):1-15.

[9] Melo MMO. Compulsão Alimentar, Imagem corporal e Qualidade de vida em crianças e adolescentes obesos [dissertação]. Belo Horizonte: Universidade Federal de Minas Gerais; 2011.

[10] Vitolo MR, Bortolini GA, Horta RL. Prevalência de compulsão alimentar entre universitárias de diferentes áreas de estudo. Rev Psiquiatr RS. 2006;28(1):20-28.

[11] Spitzer RL, Devlin M, Walsh BT, Hasin D, Wing R, Marcus M, et al. Binge eating disorder: a multisite field trial of the diagnostic criteria. Int J Eat Disord. 1992;11(3):191-203.

[12] Patsopoulos NA. A pragmatic view on pragmatic trials. Dialogues Clin Neurosci. 2011;13(2):217-224.

[13] Cooper Z, Fairburn CG, Hawker DM. Cognitivebehavioral treatment of Obesity. The Guildford Press: New York; 2003.

[14] Silva DF, Lima LL, Delfino RO, Bianchini JAA, Hintze LJ, Nardo Junior N. Efeitos de um programa multiprofissional de tratamento da obesidade e de sua cessação sobre a aptidão física relacionada á saúde de adolescentes. Rev Educ Fis/UEM. 2012;23(3):399-410.

[15] Bianchini JA, da Silva DF, Nardo CC, Carolino ID, Hernandes F, Nardo Junior N. Multidisciplinary therapy reduces risk factors for metabolic syndrome in obese adolescents. Eur J Pediatr. 2013;172(2):215-221.

[16] Cole TJ, Lobstein T. Extended international (IOTF) body mass index cut-offs for thinness, overweight and obesity. Pediatr Obes. 2012;7(4):284-294.

[17] Leite N, Lazarotto L, Cavazza JF, Lopes MFA, Bento PCB, Torres R, et al. Efeitos de exercícios aquáticos e orientação nutricional sobre a composição corporal de 
Obesidade em adolescentes e transtorno alimentar. Nardo Júnior $e t$ al.

adolescentes obesos. Rev. Bras. Cineantropom. Desempenho Hum. 2010;12(4):232-238.

[18] Heyward VH. ASEP methods recommendation: body composition assessment. J Exerc Physiol. 2001;4:1-12.

[19] Gormally J, Black S, Daston S, Rardin D. The assessment of binge eating severity among obese persons. Addict Behav. 1982; $7(1): 47-55$.

[20] Freitas S, Lopes CS, Coutinho W, Appolinario JC. Tradução e adaptação para o português da escala de compulsão alimentar periódica. Rev. Bras. Psiquiatr. 2001;23(4):215-220.

[21] Marcus MD, Wing RR, Lamparski DM. Binge eating and dietary restraint in obese patients. Addict Behav. 1985;10:163168.

[22] Johson WG, Rohan KJ, Kirk AA. Prevalence and correlates of binge eating in white and African American adolescents. Eat Behav. 2002;3:179-189.

[23] Ledoux S, Choquet M, Manfred R. Associated factors for self-reported binge eating among male and female adolescents. J Adolesc. 1993;16:75-91.

[24] Lofrano-Prado MC, Antunes HK, Prado WL, Piano A, Caranti DA, Tock L, et al. Quality of life in Brazilian obese adolescents: effects of a long-term multidisciplinary lifestyle therapy. Health Qual Life Outcomes. 2009;7:61-68.

[25] Pivetta LA, Silva RMVG. Compulsão alimentar e fatores associados em adolescentes de Cuiabá, Mato Grosso, Brasil. Cad. Saúde Públ. 2010;26(2):337-346.

[26] Souza Ferreira JE, Veiga GV. Eating disorder risk behavior in Brazilian adolescents from low socio-economic level. Appetite. 2008;51(2):249-255.

[27] Poeta LS, Duarte MFS, Giuliano ICB, Farias Junior JC. Intervenção interdisciplinar na composição corporal e em testes de aptidão física de crianças obesas. Rev. Bras. Cineantropom. Desempenho Hum. 2012;14(2):134-143.

[28] Shrewsbury VA, Nguyen B, O'Connor J, Steinbeck KS, Lee A, Hill AJ, et al. Short-term outcomes of communitybased adolescent weight management: The Loozit $\AA$ Study. BMC Pediatr. 2011;11:13.

[29] Leite N, Milano GE, Cielask F, Lopes WA, Rodacki A, Radominski RB. Effects of physical exercise and nutritional guidance on metabolic syndrome in obese adolescents. Rev. Bras. Fisioter. 2009;13(1):73-81.
[30] Coppen AM, Risser JA, Vash PD. Metabolic syndrome resolution in children and adolescents after 10 weeks of weight loss. J Cardiometab Syndr. 2008;3(4):205-210.

[31] Silva DF, Bianchini JAA, Nardo Junior N. Tratamento multiprofissional da obesidade e sua cessação em adolescentes: efeitos no perfil hemodinâmico. Motriz. 2013;19(1):195-206.

[32] Hintze LJ, Cattai GBP, Nardo Junior N. Multidisciplinary program for obesity treatment: Summary of results with adolescents. Acta Sci Health Sci. 2012;34(2):137144.

[33] Dâmaso AR, Silveira Campos RM, Caranti DA, Piano A, Fisberg M, et al. Aerobic plus resistance training was more effective in improving the visceral adiposity, metabolic profile and inflammatory markers than aerobic training in obese adolescents. J Sports Sci. 2014;32(15):1435-45.

[34] Cayres SU, Christofaro DGD, Oliveira BAP, Antunes BMM, Silveira LS, Freitas Júnior IF. Treinamento concorrente e o treinamento funcional promovem alterações benéficas na composição corporal e esteatose hepática não alcoólica de jovens obesos. Rev. Educ. Fís/UEM. 2014;25(2):285-295.

[35] Ministério da Saúde (BR). Programa de Saúde da Família. Brasília (DF); 1994.

[36] Brasil. Portaria no 164 de 24 de janeiro de 2008, Ano CXLV no 18. Diário Oficial da União; 2008.

[37] Ministério da Saúde (BR). Portaria no 425, de 19 de março de 2013. Estabelece regulamento técnico, normas e critérios para a Assistência de Alta Complexidade ao Indivíduo com Obesidade. Diário Oficial da União. 15 abr 2013; seção 1:59.

[38] Food and Agriculture Organization. The State of Food and Agriculture in 2013: food system for better nutrition [internet]. Roma [acesso em 15 fev 2015]. Disponível em: http://www.fao.org/publications.

[39] Souza L. Avaliação de Políticas e Programas de Segurança Alimentar e Nutricional no Brasil: uma análise a partir do Plano Plurianual (PPA 2012-2015). Segur. Aliment. Nutr. 2013;20(Supl):182-193.

[40] OPAS-OMS. Plano de ação para prevenção da obesidade em crianças e adolescentes. 53으 Conselho Editor, 66ํㅗ Sessão do Comitê Regional da OMS para as Américas. EUA; 2014. 